\title{
Actores, lenguas y lenguajes en el paisaje lingüístico hospitalario: entre la privatización y la agencia
}

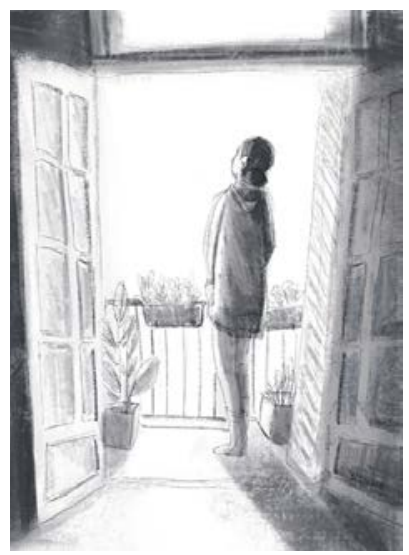

Milagros Vilar

Centro de Estudios del Lenguaje en Sociedad, CONICET -Universidad Nacional de San Martín, Argentina

milagrosvilar@gmail.com

Trabajo recibido el 1 de abril de 2019 y aprobado el 8 de junio de 2019.

\section{Resumen}

En este trabajo se presenta un estudio de caso realizado en un hospital público de la ciudad de Buenos Aires, acerca del rol de las lenguas y los lenguajes en la comunicación hospitalaria. El análisis del cruce entre los actores, las lenguas y los lenguajes nos permite observar un espacio de la comunicación en salud desregulado, en el que las instituciones se proponen monolingües y dejan buena parte de la agenda comunicacional en manos del sector privado o de las ONG. En cambio, la diversidad lingüística y cultural de la población que efectivamente concurre al hospital queda a menudo en manos de individuos que asumen el rol de mediadores entre el Estado y las comunidades.

A través de la observación directa, las entrevistas a usuarios y el relevamiento de textos multimodales disponibles en el espacio público, proponemos analizar el paisaje lingüístico del hospital desde una perspectiva etnográfica, que busca observar no solo las lenguas sino, fundamentalmente, a los hablantes y lo que ellos hacen con las lenguas y los lenguajes. Asimismo, nuestra mirada acerca de la pluralidad lingüística no se interesa exclusivamente por la diversidad de lenguas, sino también por las diferentes variedades, lectos y estilos que se observan en el hospital.

\section{Actors and language(s) in the linguistic landscape of a hospital: between privatization and agency}

\begin{abstract}
In this work we present a case study about the role of languages in healthcare communication performed at a public hospital in Buenos Aires. The analysis of the intersection between actors and language(s) shows us a communication deregulated healthcare space, where the institutions are proposed as monolingual and leave most of the communication agenda under the sphere of the private sector or non-governmental organizations.
\end{abstract}

\section{Palabras clave}

paisaje lingüístico políticas lingüísticas comunicación en salud gestión de lenguas
Keywords

linguistic landscape language policy healthcare communication language management 
In contrast, linguistic and cultural diversity of the people that actually attend the hospital often remains in the hands of individuals who assume the role of mediators between the State and the communities.

We analyze the linguistic landscape of the hospital by direct observation, interviews to patients and a collection of multimodal texts available on the public space. By using an ethnographic perspective, we aim to study not only languages but specially the speakers and what they do when they use language(s). Moreover, our perspective of linguistic plurality is not only interested in linguistic diversity but also in the different social varieties, lects and styles observed at the hospital.

\section{Atores, línguas e linguagens na paisagem linguística hospitalar: entre a privatização e a agência}

\section{Resumo}

Neste trabalho apresentamos um estudo de caso realizado em um hospital público da cidade de Buenos Aires, acerca do rol das línguas e as linguagens na comunicação hospitalar. A análise do cruzamento entre os atores e as linguagens permite observar um espaço da comunicação em saúde desregulado, onde as instituições se propõem monolíngues e deixam boa parte da agenda comunicacional nas mãos do setor privado o as ONG. Já a diversidade linguística e cultural da população que efetivamente atende-se no hospital fica nas mãos de indivíduos que assumem o rol de mediadores entre o Estado e as comunidades.

Através da observação direta, as entrevistas a usuários e a coleta de textos multimodais disponíveis no espaço público, analisamos a paisagem linguística do hospital desde uma perspectiva etnográfica, que procura observar não só as línguas, mas também, fundamentalmente, aos falantes e o que eles fazem com as línguas e as linguagens. Da mesma forma, nossa mirada acerca da pluralidade linguística não se interessa exclusivamente pela diversidade de línguas, mas também pelas diferentes variedades, letos e estilos que se observam no hospital.

\section{Introducción}

Aunque el término "paisaje lingüístico" puede remontarse a mediados de la década del noventa (cf. Landry y Burhis 1997), en los últimos diez años observamos un aumento significativo de trabajos que explícitamente se inspiran en este concepto $^{1}$. El campo de los estudios del paisaje lingüístico (Linguistic Landscape Studies, LLS) es reciente y dinámico, por lo que tanto su objeto como los modos de abordarlo han variado y se han ido diversificando a lo largo de estos años. En algunos casos, los LLS se presentan como una aproximación novedosa a los estudios del plurilingüismo, con trabajos que se dedican a registrar la diversidad de lenguas en el espacio público (Gorter 2006, Shohamy y Gorter 2008, Shohamy, Ben-Rafael y Barni 2010). Otras perspectivas conciben al paisaje lingüístico como un espacio dinámico donde se representan y negocian las identidades sociales (Blackwood, Lanza y Woldemariam 2016) y se interesan por las dinámicas de conflicto, exclusión y disidencia, a menudo provocadas por mecanismos instaurados por las políticas lingüísticas que rigen esos espacios (Rubdy y Said 2015, Moustaoui 2019, Martin Rojo 2014).

\section{Palavras-chave}

paisagem linguística políticas linguísticas comunicação em saúde gestão de línguas
1. El International Journal of Multilingualism dedicó un número monográfico al tema en 2006, y la editorial lohn Benjamins ya tiene su propia revista específica, Linguistic Landscape. An international journal, que desde 2015 ha publicado tres números por año. En el ámbito hispano, podemos mencionar las contribuciones a los estudios de paisaje lingüístico en el número monográfico de la Revista Internacional de Lingüística lberoamericana (Vol. 11 , No. 1 (21), 2013) y, más recientemente, en el número especial de la revista Lingue e Linguaggi (Vol. 25, 2018), titulado "Paisajes lingüísticos de la migración. Contextos mediáticos, urbanos y formativos", que presenta trabajos en español, italiano e inglés. 
Nuestro trabajo se beneficia de esta diversidad y se propone interrogar críticamente algunas de las nociones más extendidas en el campo de los LLS. Partimos de un enfoque que busca observar no solo las lenguas sino, fundamentalmente, a los hablantes y lo que ellos hacen cuando usan las lenguas. De este modo, nuestro estudio no pretende registrar de manera exhaustiva lo que se ve en el espacio público, sino más bien comprender el tránsito de las personas por el espacio de sus derechos, en este caso, a la salud, y analizar las distintas dinámicas entre actores, lenguas y lenguajes que contribuyen a crear el paisaje lingüístico en un hospital de la ciudad de Buenos Aires.

Desde esta perspectiva, hablamos de lenguajes para referirnos a los diferentes modos semióticos que, integrados a un repertorio relativamente estable (imágenes, gestos, posiciones, colores, sonidos, música, etc.), se combinan con el modo verbal en la producción de textos multimodales complejos (Kress y van Leeuwen 2001, Kress 2009, Jewitt 2009). Asimismo, al hablar de lenguas buscamos dar cuenta de dos formas de pluralidad lingüística que observamos en el hospital: una diversidad de lenguas y una diversidad de usos lingüísticos (variedades, lectos y estilos) que concretan estas lenguas en la vida social (Unamuno 2016). Por último, diferenciamos distintos tipos de actores que participan en el espacio hospitalario y gestionan, según diversas lógicas, la comunicación hospitalaria: actores individuales (usuarios y profesionales de la salud), institucionales (el hospital), estatales (el Gobierno de la Ciudad de Buenos Aires, el Ministerio de Salud) y otros actores (empresas y Organizaciones no Gubernamentales, ONG) que intervienen en el espacio compartido.

Desde una perspectiva teórica contextualizada en las demandas analíticas de nuestros datos heterogéneos y nuestra propia experiencia etnográfica en el campo de la salud, nos proponemos analizar cómo se configura el paisaje lingüístico hospitalario a partir de la intervención de estos actores y su articulación con diversas lenguas y lenguajes. Para ello, presentamos un abordaje etnográfico para el estudio del paisaje lingüístico a través de un estudio de caso realizado en una institución pública de la ciudad de Buenos Aires.

A partir del análisis de dos formas del paisaje (la que se presenta como visible y aquella que no está a la vista), observaremos cómo la desregulación del espacio público da lugar a nuevas dinámicas de gestión de la comunicación hospitalaria, tanto en manos de actores privados como de los propios usuarios del sistema que transitan y resignifican esos espacios.

\section{Los estudios de paisaje lingüístico}

La definición más conocida y citada, de la que parte la mayoría de los trabajos en el campo de los LLS, es la de Landry y Bourhis (1997):

the language of public road signs, advertising billboards, street names, place names, commercial shop signs, and public signs on government buildings combines to form the linguistic landscape of a given territory, region, or urban agglomeration (Landry y Bourhis 1997, 25).

Tal como señala Calvi (2018), se trata de una definición útil y potente por su sencillez, al reunir los dos elementos que constituyen la etiqueta: lo lingüístico y lo geográfico; el lenguaje y el paisaje. Otros autores, como Gorter 
(2006), simplifican al extremo esta identificación entre lo territorial y lo lingüístico, al definir al objeto de los LLS como: "languages being used on signs (hence, languages in written form) in public space" (Gorter 2006, 11).

Estas definiciones, en las que se apoya gran parte de la producción en el campo de los LLS, presentan múltiples limitaciones. Por una parte, dejan fuera de consideración un conjunto de elementos que se combinan para formar el paisaje lingüístico: las lenguas no agotan los lenguajes presentes en el espacio público, ni tampoco constituyen un código homogéneo e igual a sí mismo. Desde nuestra perspectiva, este se presenta como un espacio semiótico multimodal, cuyo estudio puede incorporar, además del modo verbal, el análisis de imágenes, gestos, posiciones, colores, sonidos, música, etcétera. En esta línea, algunos trabajos han propuesto las nociones de paisaje semiótico (cf. Jaworski y Thurlow 2010) o paisaje discursivo (cf. Bonnin 2018). Por otra parte, la idea de lenguas "en forma escrita" excluye aquellas manifestaciones verbales que forman parte del paisaje sin ser escritas. En el caso que analizamos, este aspecto adquiere gran relevancia: ciertas lenguas se vuelven "invisibles" en el espacio hospitalario, pero si prestamos atención las escuchamos y podemos observar cómo se integran y resemiotizan en el entorno en que se producen.

Las limitaciones señaladas alcanzan también a los trabajos que estudian el paisaje lingüístico en instituciones sanitarias. En general, se trata de estudios que asocian este concepto con el de accesibilidad lingüística, esto es, el grado en que un producto, servicio o entorno es accesible a los hablantes de lenguas minoritarias (Schuster, Elroy y Elmakais 2016, Schuster 2012). Estos trabajos adoptan una perspectiva institucional y se proponen medir el grado de accesibilidad de los servicios de salud según parámetros que se limitan a la presencia de las lenguas escritas (por ejemplo, la cantidad de cartelería traducida a una u otra lengua), sin considerar otro tipo de acciones que inciden en el acceso (cf. Moyer 2011).

En este trabajo, sostenemos que lo lingüístico conforma una parte -aunque fundamental- del acceso a la salud. Pero en el marco de nuestra trayectoria biográfica y social, los hablantes adquirimos competencias comunicativas ligadas no solo a las lenguas y sus contextos (Gumperz 1982) sino también competencias culturales más amplias que se desarrollan durante la práctica de actividades que las requieren ${ }^{2}$. Este proceso de desarrollo de competencias culturales permite producir e interpretar sentido a través de gestos, ropas, movimientos y nuestro propio desenvolvimiento en el espacio. Por ello, nos detendremos en otros espacios, menos explorados, que permiten el ingreso de esos otros lenguajes y formas de interacción que forman parte, también, del paisaje del hospital.

Una de las funciones del paisaje lingüístico es, justamente, organizar el tiempo y el espacio (Blommaert 2013): la modificación del ordenamiento de las sillas en el espacio institucional destinado a la espera de los pacientes; los guardapolvos blancos de los profesionales y su recorrido grupal por los pasillos del hospital; el vendedor de chipa ubicado fuera del hospital y el bar en el interior del mismo; los talleres y actividades artísticas impulsadas por organizaciones no gubernamentales; los murales que llenan de colores el exterior del hospital y las paredes blancas del interior. Todo ello nos dice mucho acerca de los diferentes modos de apropiarse o (re)construir el espacio público del hospital y la diversidad de actores y lenguajes que entran en la escena e intervienen en esa (re)construcción.
2. Blommaert (2013) denomina enskillment a este proceso de desarrollo de competencias culturales, por medio del cual se adquieren códigos, normas, criterios de inclusión y exclusión, categorías de membresía e identidades. 
Proponemos entonces abordar el paisaje lingüístico desde una perspectiva etnográfica, para comprender las regularidades, además de las irregularidades semióticas, que se presentan como una conversación de la que el observador, necesariamente, participa. Consideramos que un abordaje etnográfico del paisaje lingüístico abre el camino hacia estudios más comprensivos que permiten observar la interacción entre procesos estructurales, negociaciones entre actores locales y configuraciones espacio-temporales concretas (Blommaert y Maly 2014).

\subsection{El paisaje hospitalario en el marco de las políticas lingüísticas en salud}

Con frecuencia se vinculan los temas de paisaje lingüístico con los de política lingüística (cf. Shohamy 2015, Barni y Vedovelli 2012). En el caso que nos ocupa, es inevitable vincular ambas perspectivas debido a la naturaleza estatal de la institución hospitalaria. Se trata de un buen ejemplo para discutir las perspectivas más institucionalistas de investigación en Planificación y Política Lingüística (LPP, Linguistic Policy and Planning): los hospitales públicos serían, a priori, espacios estatales por excelencia, en los cuales deberían poder observarse las directivas del Estado en su expresión más clara. Sin embargo, como veremos, se trata de un espacio atravesado por lógicas y discursos diferentes, incluso contradictorios. En cualquier caso, en sintonía con otros trabajos realizados en el contexto argentino (Unamuno y Bonnin 2018), lo que percibimos en el hospital no es tanto una política lingüística sino su ausencia.

En efecto, consideramos que esta carencia responde a motivos ideológicos: una concepción monolingüe de los usuarios del sistema y una concepción monoglósica de la lengua (Bonnin 2015, Unamuno y Bonnin 2018). Ambas representaciones están enraizadas en uno de los principios ideológicos organizadores del Estado-nación moderno: la ecuación entre Estado, Nación, Lengua y Territorio (Glozman y Lauria 2012, Blommaert 2009). El monolingüismo público en la lengua oficial o "nacional"” $-\mathrm{y}$ el requerimiento de su uso en la comunicación pública- se da por sentado para todos los ciudadanos. De allí que, en los servicios de salud, las campañas de salud, la señalética y la comunicación con el personal médico y no médico del hospital se desarrollen exclusivamente en español. En el caso que analizamos, esta ausencia de políticas deriva en una desregulación del paisaje lingüístico que, en la práctica, permite la participación de otros actores que agencian y orientan la semiotización del espacio público, actuando como gestores del lenguaje y la comunicación en salud (Spolsky 2009).

Como punto de partida, consideramos que existe una diversidad lingüística que no es visible en el hospital en tanto no se constituye como "problema" a ser abordado por una política institucional o estatal. Esta diversidad, que no es mostrada ni forma parte de la imagen pública del hospital, emerge, sin embargo, en muchas prácticas espontáneas de actores individuales, como modos de resolver situaciones o superar barreras lingüísticas, sociales y culturales. Lo visible, en cambio, es gobernado por actores privados, que asumen funciones comunicativas propias del Estado o la institución, como la señalización del espacio o la difusión de campañas sanitarias; contribuyen, en ese sentido, a la privatización del espacio semiótico del hospital tras un estatuto de oficialidad e institucionalidad.
3. Si bien suele haber una correlación entre las lenguas oficiales y nacionales, esto no siempre es así. En el caso argentino, el castellano no posee el estatus de lengua oficial en la Constitución pero es la lengua dominante en la comunicación pública, por lo que su oficialidad es de facto. Los estados suelen reconocer una lengua como oficial cuando no hay consenso acerca de cuál es la lengua dominante, o cuando la lengua en cuestión no es, de hecho, ampliamente utilizada todavía por la población. 


\section{Presentación del caso y marco metodológico}

Con más de 140 años de antigüedad, el Hospital de Niños Dr. Ricardo Gutiérrez es uno de los hospitales generales pediátricos de referencia en el país y el que recibe la mayor cantidad de pacientes en la ciudad de Buenos Aires (un promedio de 223 pacientes por día ${ }^{4}$ ). De la población que asiste al hospital, gran parte proviene de otras provincias, con derivaciones para iniciar tratamientos específicos que no se realizan en otros centros de salud del país. Asimismo, existe una gran circulación de población extranjera, principalmente de países limítrofes y del Perú, reflejo de la distribución poblacional del Área Metropolitana de Buenos Aires, que se ha ido conformando desde mediados del siglo XX como destino privilegiado de los procesos migratorios contemporáneos. Así, en lo que respecta a la ciudad de Buenos Aires, el 38 \% de los habitantes son migrantes. Dentro de este grupo, se destaca la población proveniente del resto del país: el 12,8 \% nació en la provincia de Buenos Aires y el 12,7 \% en otras provincias. Por su parte, la población nacida en el exterior representa el 12,8 \% de la población de la ciudad y, en su mayoría, provienen de países limítrofes y del Perú $(9,6 \%)^{5}$.

Esto supone una población cuya lengua materna es una variedad del español diferente de la rioplatense o, en otros casos, una lengua que no es el español; en cualquiera de los casos, lo que se observa son repertorios lingüísticos heterogéneos, tanto monolingües como plurilingües, que contrastan con una política de aparente desconocimiento (o invisibilización) de esta diversidad por parte de la institución, con evidentes efectos desigualadores para estos colectivos migratorios (Courtis 2011), particularmente en contextos como el de salud (Jelin 2006).

Para analizar el paisaje lingüístico del hospital, recurrimos a diversos tipos de datos, obtenidos a través de un relevamiento de textos multimodales disponibles en el espacio público, entrevistas a usuarios en las salas de espera y la observación directa en el hospital. Esta última consistió en la propia observación y tránsito a través del espacio hospitalario, con vistas a identificar los diversos usos de las lenguas y los lenguajes, así como las formas de interacción y participación de los actores en el espacio. Decidimos focalizar en aquellos espacios que son transitados regularmente por los usuarios (hall de entrada, pasillos, escaleras, patios y salas de espera de múltiples servicios) y observamos también los eventos y actividades que acontecen en esos espacios, como la atención al público en la recepción, la interacción en las salas de espera y pasillos, la administración de las filas y los turnos, las actividades lúdicas o recreativas que tienen lugar en el hospital, entre otras.

En cuanto a las entrevistas, estas fueron realizadas en dos etapas. La primera se desarrolló en 2013, en el marco de un estudio exploratorio que buscaba conocer las percepciones de los usuarios acerca de la comunicación con los profesionales de salud ${ }^{6}$. La segunda etapa de entrevistas, en 2016, tuvo como propósito profundizar acerca de algunos temas de interés para este trabajo: en particular, buscábamos conocer la perspectiva de los usuarios acerca del paisaje lingüístico hospitalario. Para ambas etapas se utilizó un mismo plan de entrevista, diseñado previamente, con tres ejes temáticos: un eje sociodemográfico, orientado a conocer el perfil de la persona entrevistada (edad, sexo, lugar de nacimiento, lugar de residencia, nivel educativo, ocupación); un eje
4. Fuente: "Promedio de paciente-día en los hospitales del Gobierno de la Ciudad de Buenos Aires por especialidad según hospital", Ciudad de Buenos Aires, año 2015, Dirección General de Estadística y Censos (Ministerio de Hacienda GCBA) sobre la base de datos del Ministerio de Salud GCBA, Subgerencia Operativa Estadísticas de Salud, Ministerio de Salud GCBA.

5. Fuente: Dirección General de Estadística y Censos (Ministerio de Hacienda GCBA), EAH 2015.

6. En este estudio participaron también Juan Eduardo Bonnin, Luz Espain y Geraldina Fernández Tuñón, quienes formaron parte del equipo que llevó adelante el diseño y la recolección de las entrevistas. Algunos de los resultados de esta primera etapa de investigación pueden encontrarse en Vilar y Espain (2016). 
sociolingüístico, en el que se indagaba acerca del conocimiento de lenguas y sus ámbitos de uso; y un eje comunicativo, a través del cual buscábamos conocer las concepciones de los usuarios acerca de las prácticas comunicativas en el hospital ${ }^{7}$. En total, se recolectaron 13 entrevistas a pacientes, familiares y acompañantes en las salas de espera del hospital.

A continuación, proponemos un recorrido posible por el paisaje lingüístico de un hospital de la ciudad de Buenos Aires, para conocer la diversidad lenguas, lenguajes y actores que están presentes y se articulan para configurar el espacio público. Analizaremos, en primer lugar, el paisaje visible, aquel que se muestra dominado por el monolingüismo español y se presenta, bajo diversas formas y materialidades, en el modo escrito. A continuación, nos detendremos en el paisaje invisible, conformado por otras formas de diversidad lingüística que, asociadas a otros territorios y tiempos, circulan también por determinados espacios del hospital, generando dinámicas específicas con actores que funcionan como mediadores entre espacios y lenguas.

\section{El paisaje visible: privatización, polifonía y temporalidades en conflicto}

En el caso del paisaje visible ${ }^{8}$, es decir, de aquel que se manifiesta a través de distintas formas y materialidades de la escritura en el espacio público, observamos una tensión que, siempre en torno al español, nos permite comprender la diversidad de actores y relaciones que participan en su configuración.

Desde el punto de vista de la materialidad, podemos trazar un continuum que va del cartel offset, diseñado e impreso profesionalmente, hasta la nota manuscrita pegada de manera precaria. Desde el punto de vista de su funcionamiento discursivo, podemos establecer distintos grados de dependencia espacio-temporal. El offset está diseñado para perdurar en el tiempo: aun cuando luego puedan sumarse nuevas capas semióticas, éstas lo modifican pero no lo anulan. Algunos carteles de este tipo tienen un grado más alto de independencia espacio-temporal: el anuncio de servicios ("BA Wi-Fi") o normativa general ("Prohibido fumar") no solo persisten en el tiempo sino que son relativamente independientes en el espacio; su campo de aplicación se extiende a todo el entorno institucional, no solo al lugar en el que están colocados. El cartel manuscrito, en cambio, es el más efímero; su propia materialidad sugiere espontaneidad: "Vuelvo más tarde" es tan deíctico y contextualmente dependiente como pueda serlo, tanto en el aspecto espacial (vuelvo a este lugar preciso en el que el cartel está pegado) como en el temporal (más tarde en el día de hoy).

\subsection{Una institucionalidad aditiva}

El espacio es una construcción que, producida socialmente, también está atravesada por el tiempo; en tal sentido, el paisaje lingüístico no solo es producto de procesos que se desarrollan en el tiempo (y se observan en el espacio), sino que también muestra las huellas de esos procesos. En la Imagen $1^{9}$ pueden observarse las dinámicas históricas de interacción entre actores y acciones en el paisaje.
7. Para el presente trabajo, nos detuvimos especialmente en algunas preguntas de estos ejes: en cuanto al sociolingüístico, nos centramos en las preguntas acerca de la comunicación oral (por ejemplo: ¿Habla otras lenguas? ¿Con quién/ es? ¿En el ámbito del hospital? ¿Por qué usa una lengua u otra? ¿Para qué? ¿Escuchó hablar otras lenguas en el hospital? ¿A quién/es?); y, en cuanto al eje comunicativo, aquellas que trataban acerca de la comunicación con los profesionales de la salud en la consulta médica (por ejemplo: ¿Diría que hay / no hay "buena comunicación" con los médicos? ¿En qué consistiría? ¿Recuerda ejemplos de buena y mala comunicación?) y sobre las prácticas de lectura en el hospital (¿Suele leer los carteles/ las carteleras que hay en el hospital? ¿Por qué? ¿De qué temas tratan?).

8. El análisis se centrará en los espacios del hospital que fueron relevados mediante la observación directa, ya mencionados en el apartado 3, esto es, aquellos que son transitados por los usuarios cuando asisten al hospital: hall de entrada, pasillos, escaleras, salas de espera y patios.

9. Todas las fotografías incluidas en este trabajo fueron tomadas por la autora en el Hospital de Niños “Dr. Ricardo Gutiérrez" de la Ciudad Autónoma de Buenos Aires entre 2014 y 2016. 


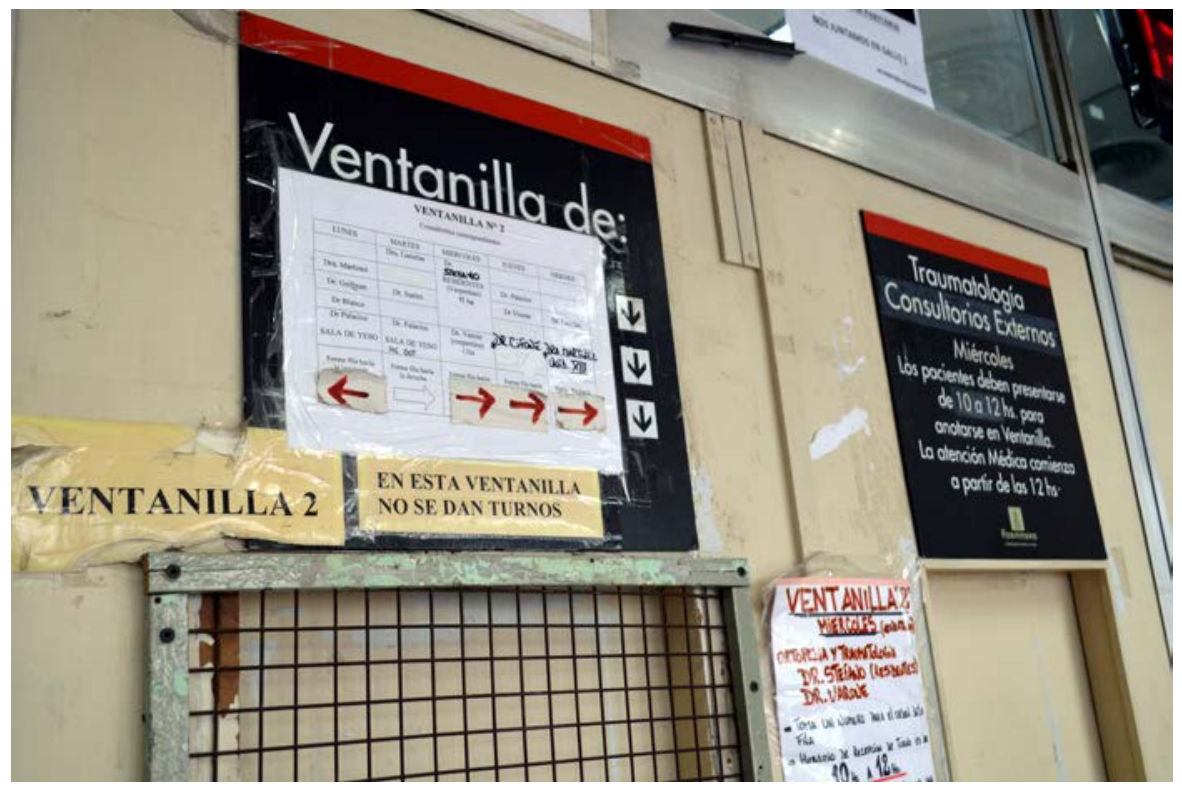

Imagen 1. Cartelería en el sector de consultorios externos del servicio de Traumatología.

En primer lugar, se observa la señalética impresa en offset, fruto de una donación de un laboratorio privado, Roemmers, que tuvo a su cargo la función de señalización del espacio en prácticamente todo el hospital. Su presencia genera un efecto de identificación entre el espacio señalado y la empresa, tanto por el uso de los colores y la tipografía que forman parte de la identidad institucional, como por la presencia del logotipo y el eslogan ("Conciencia por la vida") al pie de los carteles, prácticamente como una firma. Habría, entonces, cierto efecto de privatización de la semiosis del espacio hospitalario, cuyo tránsito es organizado por el laboratorio.

Ahora bien, el efecto de durabilidad y permanencia que transmite la señalética donada por el laboratorio Roemmers muestra su caducidad cuando diversas voces y temporalidades entran en conflicto en el paisaje. En efecto, la fotografía muestra una serie de interacciones: al cartel en offset que señalaba un lugar y tres ítems, ahora ilegibles, se le adiciona un segundo cartel, impreso en papel y tinta negra, fijado con cinta adhesiva transparente. A diferencia del primero, aquí se emplea papel blanco, de bajo gramaje, con una tipografía estándar negra y sin firma. Se trata de un cartel ostensiblemente barato, menos formalizado e institucional, que modifica la información acerca de la distribución de las ventanillas de atención a los usuarios. Del mismo modo, este cartel también pierde actualidad y es modificado con correcciones manuscritas, que no solo cambian los nombres de los profesionales que atienden sino también la redistribución de las filas correspondientes a cada consultorio.

En la parte inferior, otros dos carteles vuelven a resignificar la ventanilla, que ya no se define por un sintagma preposicional ("ventanilla de") sino por un número, siguiendo un modo habitual de organización del espacio institucional ("ventanilla 2"). Probablemente contemporáneo, como evidencia el color amarillento que ha adquirido el papel, a su lado hay otro cartel que indica las acciones no disponibles para realizar en esa ventanilla: "dar turnos". Ahora bien, ninguno de esos carteles dice qué es lo que se hace en la ventanilla 2: allí no se dan turnos, pero de alguna manera se administra el acceso a los consultorios y se indica incluso hacia dónde debe formarse 
fila. Para saber qué es lo que se hace en ese espacio o para qué hay que formar fila, es necesario recurrir a otros medios, como la propia experiencia o el consejo de alguien más experimentado, como un mediador.

El gesto de dejar por escrito aquello que se transmite de manera recurrente en una interacción ("en esta ventanilla no se dan turnos") se presenta como un intento de actores individuales (en este caso, el personal administrativo) de capturar el discurso transitorio y efímero, garantizándole cierto grado de fijeza y permanencia en el espacio. Vemos aquí cómo determinados agentes, probablemente no autorizados para asumir tareas tales como la comunicación institucional, actúan de modo tal que su voz individual pasa a tener, a partir de la escritura y la instalación del cartel en el espacio público, un estatuto institucional. A partir de esta forma particular de entextualización (Briggs y Bauman 1992), podemos ver que lo que era una intervención individual y espontánea sobre el espacio asume un carácter institucional y es utilizada como un modo (aunque informal) de ejercer la autoridad de la institución (Park y Bucholtz 2009), regulando las conductas de los individuos que transitan en ella.

En efecto, la superposición de carteles no pierde cierto efecto de institucionalidad: ninguno tapa por completo al anterior, sino que lo modifica. Con mayor o menor grado de formalidad, en esos carteles la voz autorizada sigue correspondiendo a la institución, capaz de definir las conductas y regulaciones y de disciplinar a los sujetos que transitan ese espacio. Esta comunicación "en capas" que se observa en el espacio físico del hospital habilita una lectura diacrónica acerca de la dinámica funcional de la institución, que cambia de nombres, usos y conductas constantemente. Eso es visible no solo en las formas escritas de la lengua sino también en las interacciones orales pasadas que dejan sus huellas y pueden leerse allí, como las preguntas de los pacientes que hacen que en la ventanilla 2 se pegue un cartel que indique que allí no se dan turnos.

En tal sentido, podemos afirmar que no hay un único paisaje visible en el hospital sino que existen diferentes estratos comunicativos que se producen en escalas diferentes e interconectadas, generando dinámicas espacio-temporales particulares: el pedido recurrente de turnos en el lugar equivocado da lugar a una necesidad del personal, que se manifiesta en la intervención concreta en el espacio, mediante un cartel que dialoga, a su vez, con otro que ya estaba allí. En este proceso, el espacio es semióticamente co-creado y formado por diferentes agentes en una interacción compleja.

\subsubsection{Dinámicas de interacción en el paisaje}

A partir del ejemplo analizado, podemos distinguir dos formas de comunicación que conviven en el paisaje lingüístico hospitalario: por un lado, la comunicación representada por la cartelería en offset realizada por diferentes entidades, con un predominio notable de la gestión privada en manos de laboratorios (Roemmers) y otras empresas (San Up), que produce un efecto de identificación de estos actores privados con la institución. Y, por el otro, una forma de comunicación más espontánea, representada por aquellas respuestas locales a las necesidades de los que transitan por aquellos espacios, esto es, trabajadores y usuarios del hospital.

A partir de lo observado, no hemos podido identificar una voz institucional claramente definida ni tampoco una identidad asociada a ésta (por 
ejemplo, a partir del uso de un logotipo, determinada tipografía y colores, etc.). Más bien, la institución aparece en el paisaje como una suma de iniciativas - en apariencia desconectadas - de agentes institucionales anónimos, que se apoyan en la comunicación planificada por los actores privados y la resignifican. Podemos pensar esta dinámica interactiva en los términos que utiliza Goffman (1981) para describir las distintas estructuras de participación en las interacciones cara a cara: muchas veces se generan, de manera simultánea a la comunicación principal o dominante, otras formas de comunicación entre los participantes, que constituyen aquello que denomina "comunicación subordinada", es decir, aquella que "es alentada, entonada y sincronizada para que constituya una perceptible interferencia que atenúe lo que podría denominarse la comunicación dominante en su entorno inmediato" (Goffman 1981, 133). Trasladándolo al caso analizado, observamos una jerarquización del paisaje lingüístico, donde es posible distinguir entre una comunicación principal -cartelería en offset planificada por agentes privados-y una subordinada, gestionada por actores institucionales anónimos, que surge en respuesta a la primera y con funciones comunicativas diferentes, como veremos enseguida.

A partir del año 2016 hemos observado un trabajo de semiotización del espacio hospitalario por parte del Estado, el Gobierno de la Ciudad de Buenos Aires. Aunque se encuentra en los centros de salud desplegados en el territorio, que son nuevos y tienen, entonces, toda la señalética diseñada profesionalmente, en los hospitales ya existentes se introduce lentamente. Por una parte, se instalaron rápidamente los carteles de servicios e identidad gubernamental, como "BA Wi-Fi" (Imagen 2); por otra parte, la señalética destinada a organizar el espacio también comienza a verse, aun cuando su contenido deba ser igualmente completado o acentuado mediante cartelería de segunda mano, como sucede en el sector más moderno del hospital (Imagen 3).

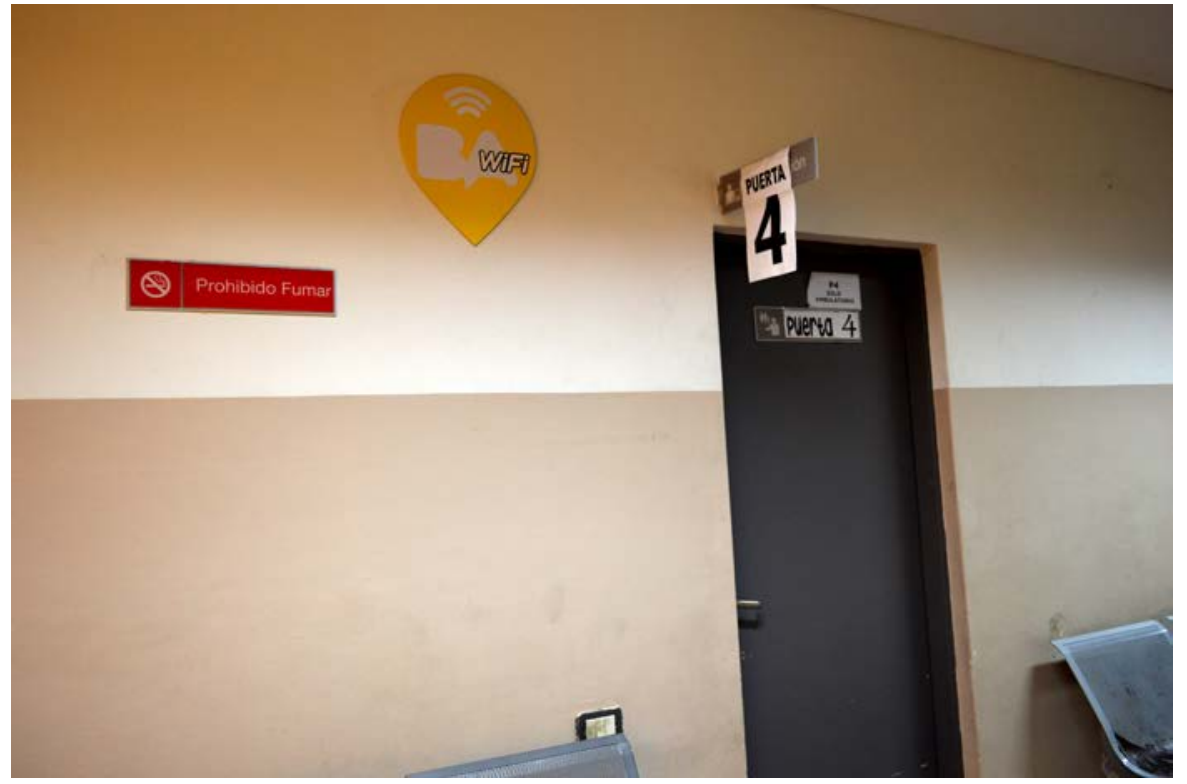

Imagen 2. Carteles de diferentes temporalidades en un pasillo de la planta baja. 

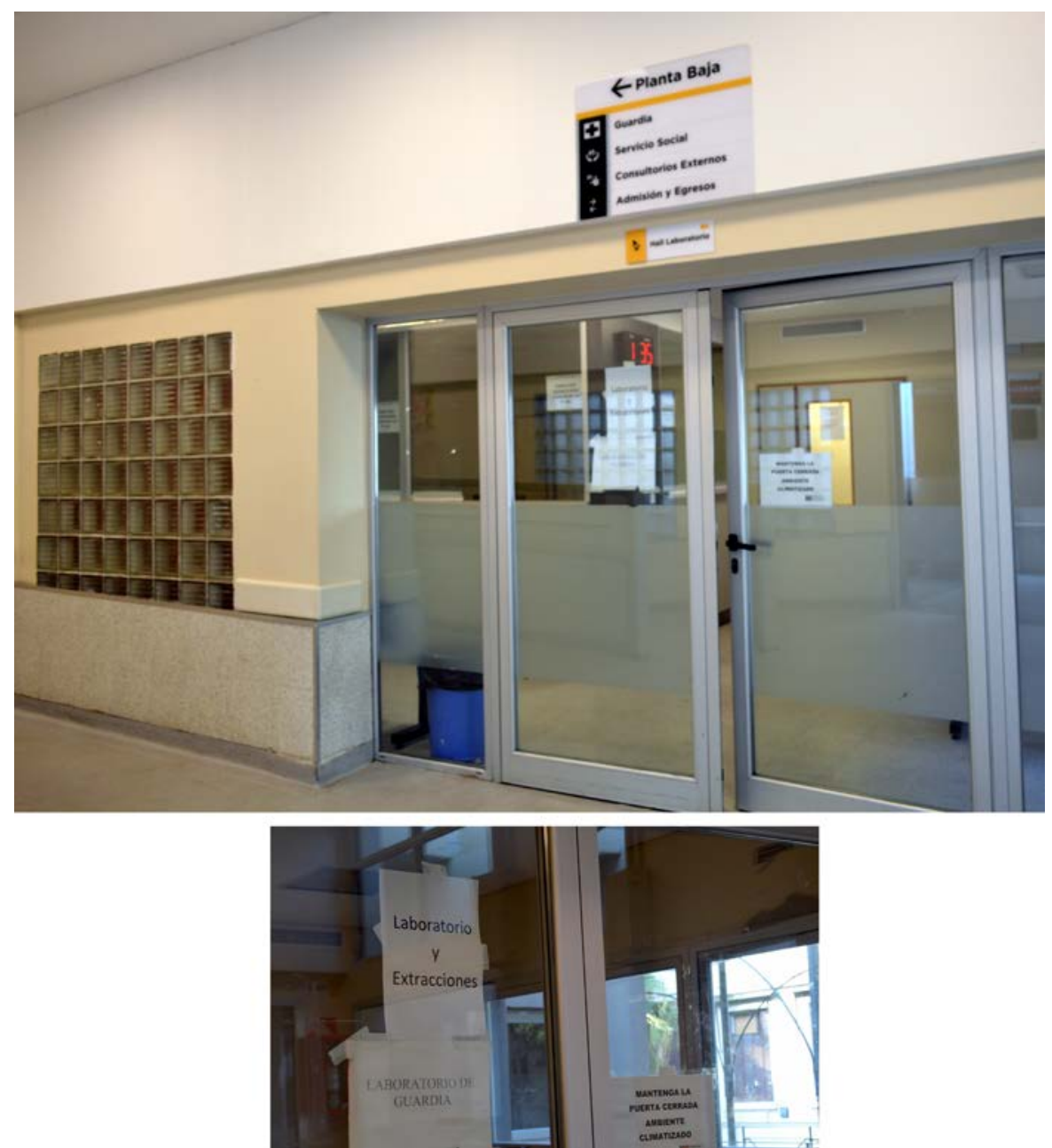

Imagen 3. Sector de Laboratorio en la planta baja del hospital.

Los modos de comunicación subordinada establecen diferentes tipos de relaciones con la comunicación principal: pueden complementarla, corregirla, incluso interferir en ella, pero no la detienen. Como vimos en la Imagen 1, el cartel de Roemmers todavía está ahí, aun cuando su contenido haya quedado reducido al mínimo (apenas indica "esta es una ventanilla": no sabemos cuál, ni qué función tiene) y se reitere en posteriores carteles. Las necesidades que habilitan esta comunicación varían, desde la actualización o corrección de información que es considerada antigua o incorrecta (Imágenes 1 y 2) o la complementación de esta comunicación mediante información más detallada (Imagen 3) hasta la introducción de nueva información que no se encuentra en la cartelería oficial ("no se dan turnos", "entre sin golpear", "pasar con orden en mano", etc.).

En la comunicación principal predominan las funciones comunicativas de señalización del espacio, de información acerca de los servicios (Wi-Fi) y otros aspectos generales de la institución (lo que respecta a la seguridad del edificio, planos de evacuación, residuos, etc.), o bien regulaciones o prohibiciones también generales, como el cartel de "prohibido fumar" (Imagen 2). Presentadas mediante una voz pública que reduce al mínimo las huellas de la subjetividad, estos mensajes tienen un alto grado de independencia espacio-temporal y no muestran las tensiones que señalamos en la Imagen 1. 
En un grupo aparte están aquellos carteles que informan acerca de campañas como la de vacunación, donación de sangre y prevención de enfermedades. En ellos se hacen presentes, además de los agentes privados, ciertos agentes estatales, como el Ministerio de Salud: por ejemplo, en las campañas contra el dengue, la fiebre chikungunya y el zika, que vienen siendo intensamente difundidas desde 2015 y cuyo afiche encontramos en un lugar muy poco estratégico del hospital, como es el contorno de la puerta de ingreso a la oficina de Recursos Humanos, junto a una gran cantidad de carteles destinados al personal de salud (Imagen 4).

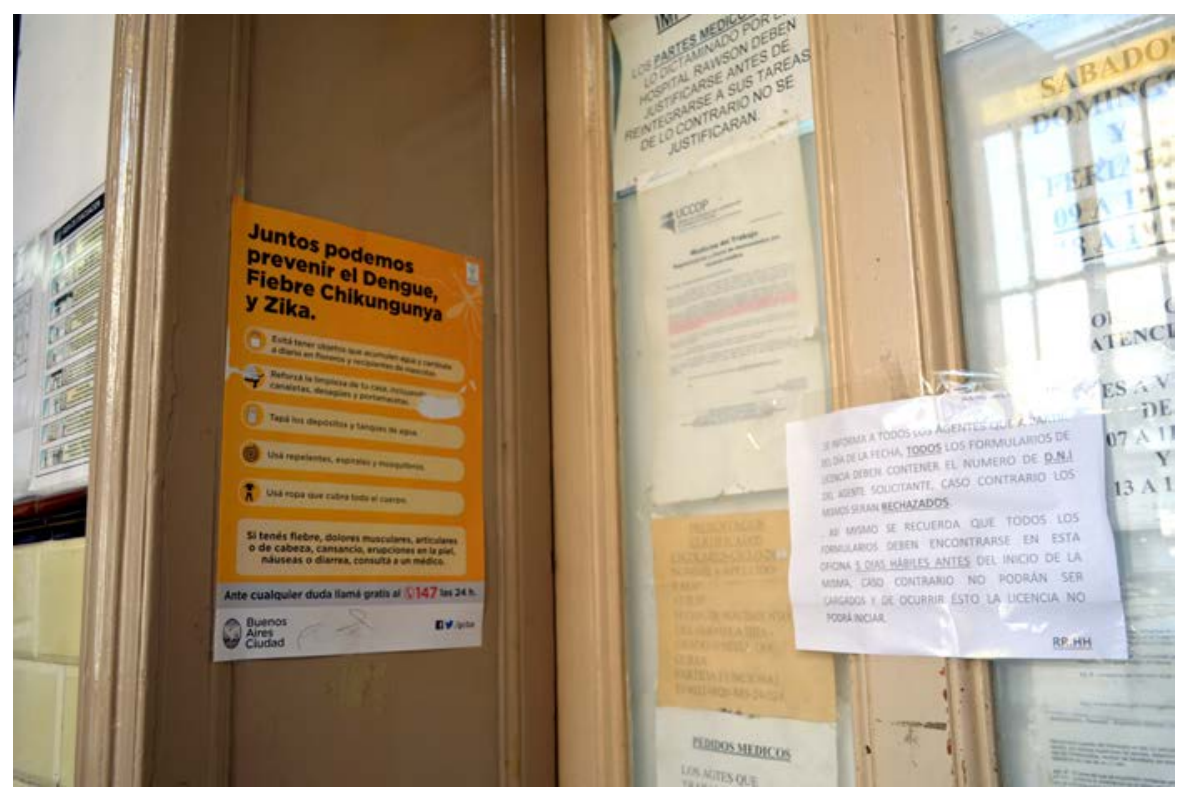

Imagen 4. Afiche de prevención del Gobierno de la Ciudad de Buenos Aires colocado en el sector de Recursos Humanos del hospital.

La función comunicativa predominante en la comunicación subordinada es, en cambio, la instrucción: los carteles, impresos o manuscritos, con distintos grados de cortesía, señalan normas, procedimientos, modos de comportarse en diferentes instancias del hospital. En general, aportan datos o información adicional que se considera faltante en la comunicación dominante y que se orienta al desarrollo de una tarea específica, tanto de carácter administrativo (dónde formar fila) como sanitario (quiénes deben vacunarse). Esto último puede verse en la información que se agrega de manera manuscrita, con marcador de color rojo, en el afiche sobre vacunación del Ministerio de Salud (Imagen 5). Por lo tanto, cabe señalar que su carácter relativamente espontáneo o su modo de instalación precario no implica que el contenido vehiculizado sea secundario o menos importante; al contrario, suele ser fundamental para los usuarios y, sobre todo, para los menos experimentados: indica lo que pueden o no hacer y cómo deben hacerlo. En todo caso, que esta comunicación tenga un lugar subordinado en el espacio nos habla otra vez acerca de la escasa planificación y gestión del paisaje lingüístico por parte de actores gubernamentales, que terminan dejando en manos de actores privados el diseño de la señalética hospitalaria o dependiendo de la iniciativa del personal del hospital. 




Imagen 5. Afiche del Ministerio de Salud de la Nación sobre vacunas, colocado en la puerta de entrada a un consultorio en el sector de Vacunación.

\subsection{El paisaje visto desde el usuario}

Hasta aquí, nos hemos enfocado en cómo se han ido generando estas capas de la historicidad en el paisaje lingüístico del hospital y sus diferentes modos de interacción, pero no hemos indagado acerca de qué es lo que los propios actores, aquellos que recorren el espacio, ven en él. Nos centraremos en aquellos que forman parte de la instancia de recepción de la mayoría de los carteles presentados: los usuarios. A modo de exploración de lo que sucede en la recepción, entrevistamos a algunos usuarios para saber si leían los afiches y carteleras del hospital y qué información encontraban allí. Algunos 
señalaron una lectura asociada a un fin específico: "cuando busco algo" (entrevista 1, 2016) o cuando se presenta alguna necesidad, como la de saber "para dónde tengo que ir" (entrevista 5, 2016). La cartelería es inseparable de una de sus funciones, aquella que hemos señalado como predominante en la comunicación principal, la de señalamiento del espacio: "[los carteles] te indican dónde está el consultorio" (entrevista 1, 2016). El uso de estos carteles se vincula, entonces, a un conocimiento del funcionamiento del hospital y del espacio físico: así, una entrevistada dice no prestarles atención debido a que "ya conozco el hospital y entonces generalmente me comunico con la doctora o con la secretaria" (entrevista 2, 2016).

En este sentido, podríamos pensar que la comunicación que hemos caracterizado como "subordinada", asociada a una función instruccional, no aparece diferenciada de esa manera para los usuarios; más bien, que hayan referido a las funciones de comunicación "oficial" no quiere decir que ignoren lo que está a su alrededor, sino que refuerza la idea que habíamos mencionado acerca del efecto de institucionalidad que tienen, también, los carteles colocados espontáneamente por actores individuales.

Finalmente, observamos también que la existencia de carteles que indican los procedimientos para obtener turnos o la ubicación de los consultorios no resulta suficiente para algunos usuarios, que igualmente recurren a otras personas para informarse, como la secretaria o los mismos médicos. Incluso hemos notado que con frecuencia preguntan a otros usuarios sobre cómo realizar determinados procedimientos o cómo llegar a ciertos lugares ${ }^{10}$.

\section{El paisaje invisible: lenguas de otro lugar y otro tiempo}

Algunos estudios de paisaje lingüístico realizados en contextos plurilingües argumentan que ciertos patrones de distribución de las lenguas presentes en el espacio público pueden funcionar como un índice de los repertorios lingüísticos de las personas que recorren esos espacios (Soler-Carbonell 2016). Desde esta perspectiva, la población que asiste al hospital tendría repertorios monolingües, ya que, si nos guiamos por lo que está a la vista -esto es, lo escrito-, el español es la única lengua presente en el paisaje. Si bien existieron campañas gráficas del Ministerio de Salud de la Nación que incorporaron traducciones a lenguas indígenas, como es el caso de la campaña de prevención del cáncer de cuello de útero, disponible en guaraní, toba y wichi ${ }^{11}$, estos afiches no se encuentran en el hospital de la ciudad de Buenos Aires.

Sería errado decir que las lenguas minoritarias no forman parte del paisaje lingüístico del hospital, dado que están presentes en él sin ser escritas: no se ven, pero sí pueden oírse, especialmente si las buscamos. Las lenguas indígenas no aparecen, aquí, en intervenciones escritas de los usuarios y mucho menos de los profesionales; sin embargo, ingresan en el hospital y tienen una circulación que difiere de la lengua del Estado, el español.

Así como los diferentes modos de comunicación que se podían observar en la señalética del hospital, vemos que existen aquí lenguas que se usan en determinados circuitos formales de la institución (la administración, la consulta médica, la ventanilla de informes, etc.) y otras lenguas, con un ámbito de uso más restringido en el hospital. Estas últimas pueden escucharse en las salas de espera, algunos pasillos, el patio exterior, pero se dejan de
10. En este punto, hemos encontrado muchas similitudes con un estudio realizado por el Programa de Mejora de la Atención a la Comunidad del Ministerio de Salud de la Provincia de Buenos Aires acerca de la atención al usuario en tres hospitales públicos de la provincia (Remorini et al. 2007). En el apartado sobre información al usuario, los resultados del relevamiento señalan aspectos muy similares a los observados en el hospital de CABA: "Si bien en algunos servicios de estos hospitales hemos notado la existencia de carteles indicando los procedimientos para obtener turnos o lograr la atención, su visualización resulta en ocasiones dificultosa por la cantidad y superposición de los mismos en algunos sectores. Asimismo, registramos que la mayoría de las personas luego de haber leído los carteles, igualmente recurren al personal para evacuar dudas o para confirmar que la información contenida en los mismos "continúa vigente" o incluye "todo lo que se necesita saber", en función de experiencias pasadas de inconsistencia entre lo expresado en los carteles y lo efectivamente solicitado al usuario. Asimismo, en un alto porcentaje, los usuarios además de consultar al perso nal, preguntan luego a otros usuarios sobre cómo procedieron ellos mismos, porque en ocasiones las explicaciones de los primeros no resultan demasiado claras" (Remorini et al. 2007, 56).

11. Por ejemplo: <http://www. msal.gob.ar/images/stories/ryc/ graficos/ooooooo774cnt-21-aficheVPH_misiones guarani-cast_2016. pdf $>$ [acceso el 15/03/2019] 
oír al entrar al consultorio o al acercarnos al mostrador de informes. La frontera entre estas lenguas está más o menos marcada por el ingreso de los individuos a determinados espacios, como lo ilustra la siguiente escena observada: dos personas llegan al hospital hablando guaraní, pasan la puerta de entrada y alternan al español.

\subsection{Espacios que delimitan lenguas}

Ciertos espacios condicionan la organización de los patrones de plurilingüismo y explican fenómenos de alternancia de código (Fernández Vavrik 2012) como el observado en la puerta del hospital. Por ello, proponemos hacer una distinción entre lo que llamamos "lenguas de interior" y "lenguas de exterior". En este caso, el interior está claramente orientado hacia el monolingüismo: tanto en la consulta con el médico como en la administración, en las carteleras y en otros documentos escritos, como formularios, recetas médicas y, como vimos, señalética, la norma que subyace es "solo español". Las lenguas de exterior, en cambio, circulan en espacios liminares (Rampton 1997), esto es, aquellos que están situados en una zona de transición entre el adentro y el afuera de la institución (como los patios y pasillos) o los que permiten una mayor apropiación por parte de los usuarios, como ocurre con las salas de espera. Así, por ejemplo, vemos cómo se organiza una sala de espera como una unidad espaciotemporal habitada por múltiples comunidades, delimitadas especialmente por bolsos, ropa y disposición de las sillas, cuando son móviles. En esa especial configuración del entorno, las lenguas no se ven, pero se oyen y son reconocidas por otros hablantes:

(1)

MV: ¿Y acá en el hospital escuchaste hablar otras lenguas?

EA: Sí, he visto (inaudible) otros hablan aymara, otros hablan quechua, otros hablan guaraní también

MV: Ah, ¿y cómo las reconociste a las lenguas, porque algo sabés?

EA: Del aymara sé algo y del quechua no, o sea...

$\mathrm{MV}$ : Podés reconocerla si escuchás hablar...

EA: Sí, sí, sí, la puedo reconocer...

(Entrevista 4, marzo de 2016)

El interior del hospital es un espacio donde se reconocen lenguas pero donde pocos se reconocen como hablantes de esas lenguas. En el fragmento (1), la entrevistada de origen peruano - que había declarado hablar "solo español" - no se reconoce como hablante bilingüe pese a tener la capacidad de escuchar esas otras lenguas y reconocerlas. Este hecho se vincula a lo que han señalado algunos estudios (cf. Gandulfo 2012), respecto a que los hablantes con repertorios plurilingües a menudo pueden sentirse incapacitados comunicativamente cuando se encuentran "fuera de lugar". El valor y la función de los recursos lingüísticos de los hablantes adquieren otros significados en un espacio público como el hospital, que impone determinados usos de lenguaje. Incluso lo que significa "hablar una lengua" varía según la situación (Vilar 2016) y la representación del propio saber lingüístico se modifica, como vemos en la expresión "del aymara sé algo" de la entrevistada en el fragmento anterior.

Estas lenguas minoritarias, "lenguas de exterior", o bien son ocultadas por los hablantes o bien representadas como lenguas que pertenecen -justamente- a otros espacios y otros tiempos: la infancia, el lugar de origen, el entorno familiar: 
(2)

JB: Le hago una pregunta... ¿Usted habla otras lenguas además del español?

EA: No

JB: ¿Ahí en Corrientes no hablaban guaraní?

EA: Nah, ya está, pasó ya eso...

JB: ¿Por qué ya pasó?

EA: Y porque yo no me crié en Corrientes, soy nacida, y a los catorce años me vine a

Buenos Aires

(Entrevista 8, junio de 2013)

(3)

JB: Y vos, cuando hablás allá con tus paisanos, ¿sobre qué temas hablás?

EA: Nosotros...

JB: Porque vos evidentemente hablás perfecto castellano...

EA: Claro, sí, y yo... Y hablamos también sobre... O sea que por ahí, con mis paisanos, por ahí llegaron de allá y les cuento de acá, esas cosas, para tenerlos más al día de las cosas de aquí. Mayormente hablo en quechua a las personas que llegan de allá y por ahí no se adaptan mucho, entonces, como yo... Mi mamá nos crió así, sin olvidarnos del habla del quechua, entonces yo hablo, hablo con las personas mayores más que todo

JB: Con las personas mayores sobre todo

EA: Sí

(Entrevista 2, junio de 2013)

En (2), el guaraní está asociado a un espacio lejano ("allá": la provincia de Corrientes) y a un tiempo pasado (su infancia, que "ya pasó"), que constituyen los motivos por los cuales la entrevistada no declara ser hablante de esta lengua: el paso del tiempo y el cambio de lugar hacen que "pierda", de alguna manera, la lengua. En cambio, en el fragmento (3), la entrevistada, de origen boliviano, se reconoce como quechuahablante y dice haber sido criada para no olvidar su lengua; en este caso, el quechua está asociado al ámbito familiar y a una función de conexión entre el país de origen y el de destino. Los hablantes bilingües adquieren así una función de "puente" entre dos espacios, como mediadores entre dos lenguas (de interior y de exterior).

\subsection{Entre espacios, lenguas y variedades: los mediadores}

Ya hemos observado que la desregulación del espacio público por parte del Estado da lugar a la gestión local y propicia la emergencia de nuevos actores que intervienen en la planificación de las prácticas de comunicación, como el personal que modifica la cartelería que se encuentra desactualizada o incompleta. Si nos limitamos a las interacciones escritas que observamos en el hospital, solo vemos una parte del paisaje: debemos pensar esa interacción que vimos en la Imagen 1 en relación con otras interacciones que se producen en el entorno hospitalario. Lo hemos experimentado al transitar los pasillos del hospital o al detenernos en alguna sala de espera: muchas personas se han acercado a pedirnos algún tipo de información. Si no somos trabajadores del hospital ni usuarios frecuentes, es poco probable que podamos ayudar, ya que se trata de un conocimiento adquirido por la experiencia de estar allí, un saber práctico al que no se accede simplemente leyendo los carteles. Quienes poseen este saber práctico saben cómo transitar el espacio del hospital, ya han adquirido una experiencia en ello, por lo que pueden guiar y acompañar a otros que lo necesitan.

Varios trabajos han señalado la presencia de mediadores o intérpretes casuales en los servicios de salud de la ciudad como un modo de superar 
los obstáculos de la comunicación entre usuarios y profesionales (Jelin 2006, Finkelstein 2017, Vilar 2018). Se trata de hablantes bilingües, como la entrevistada hablante de quechua del fragmento (3), que funcionan como mediadores lingüísticos y culturales con otros usuarios, como los "recién llegados" o las personas mayores, cuyo conocimiento del español rioplatense, en particular, y del sistema de salud, en general, es más limitado. Estos mediadores suelen ser gestionados por las propias comunidades, pero en ocasiones son también los trabajadores de la salud quienes deben acudir a otras personas para que funcionen como intérpretes ad hoc ${ }^{12}$.

Otro tipo de mediador que emerge del análisis de nuestras entrevistas es aquel que realiza una traducción del lenguaje especializado de la Medicina a una variedad que pretende ser comprensible para todos. Ya sea para despejar dudas, para lograr una mejor comprensión o para explicar a otros, quienes pueden ocupar este rol son personas del entorno del paciente o familiar que tienen una formación o trayectoria profesional asociada al ámbito médico.

Aunque en la consulta médica se hable solo español, las dificultades en la interacción son frecuentes, lo que prueba que no alcanza con compartir el código de la lengua para comunicarse eficaz y plenamente. La siguiente entrevistada, una mujer argentina de 45 años, está con su hija en el hospital y señala que en la consulta médica está acompañada porque su hermana es médica. Esto le brinda una mayor seguridad en el proceso de atención médica, que justifica con la falta de saber: "si no sabés, tal vez no te sentís acompañada y por ahí le pifian".



Nuevamente, observamos un modo particular de resolver los problemas de comunicación con que se enfrentan los usuarios cotidianamente, que surge de manera espontánea. A diferencia de un tipo de gestión planificada desde la institución o el Estado, observamos aquí aquello que
12. Hemos tomado conocimiento de casos en que se acudió a otros pacientes, familiares (a veces incluso niños), o personal bilingüe que trabaja en el hospital para que oficien de intérpretes en las consultas. En la literatura sobre mediación lingüística en salud se han estudiado las consecuencias negativas del uso de intérpretes no profesionales y su impacto en la calidad de la atención (para una extensa revisión, cf. Flores 2005). 
Louis-Jean Calvet (1997) en su clásico libro había definido como "gestión in vivo", un tipo de solución que tiene su origen en las prácticas sociales, contrastándola con la "gestión in vitro", que supone una intervención en sentido opuesto y con un mayor grado de planificación. La señalización del espacio, como vimos, muestra huellas de una gestión planificada, en manos de los laboratorios, pero que con el paso del tiempo se fue alejando de las prácticas reales; allí, los mismos actores fueron modificando los carteles, es decir, gestionaron in vivo la señalización del espacio para solucionar los problemas que fueron encontrando. La figura del mediador o acompañante es también un modo de enfrentar ciertos obstáculos en las prácticas, ligados al desconocimiento de las lenguas, variedades y lenguajes dominantes en una institución que, como muchas otras, exige el conocimiento de ciertas reglas y que, en el caso del hospital, puede llegar a delimitar quiénes tienen acceso o no a la salud. De este modo, los actores locales resignificaron las acciones de comunicación diseñadas por empresas privadas o el gobierno de la ciudad: al intervenir la cartelería o actuar como mediadores entre lenguas ponen de manifiesto los límites de estas políticas monolingües, que no siempre son coherentes con las necesidades de los profesionales y usuarios de la salud.

\section{Actores y acciones del paisaje lingüístico hospitalario: conclusiones}

Observar el paisaje lingüístico hospitalario desde una perspectiva etnográfica nos ha permitido realizar más que un registro exterior de lo que se ve en las paredes públicas de la institución. Nos hemos interrogado acerca del acceso a los derechos de los usuarios en el ámbito de la salud y hemos podido ver cómo estos derechos se ven amenazados por una institución que se presenta como monolingüe y un Estado ausente en la formulación y sostenimiento de políticas lingüísticas y comunicacionales en salud. Ante esta amenaza, la gestión local se presenta como una alternativa al ocultamiento o invisibilización de los problemas que deben enfrentar los individuos (ya sean usuarios o trabajadores de la salud) en sus prácticas cotidianas, no solo para acceder a sus derechos sino también para poder garantizarlos. Los obstáculos abarcan mucho más que el pretendido monolingüismo de la institución: ¿̇cómo ubicarse y transitar un espacio repleto de indicaciones superpuestas? ¿Cómo interpretar los procedimientos para acceder a un turno o realizarse un estudio, si estos son opacos, incompletos o se encuentran desactualizados? Y una vez superados estos, nuevos obstáculos aparecen en la instancia de interacción con los profesionales médicos, que exigen muchas veces la intermediación bajo la forma de traducción intralingüística hacia un lenguaje más accesible.

Como suele suceder, ante la ausencia del Estado, la gestión del paisaje lingüístico queda en manos de actores privados. Estos constituyen una alternativa tendiente a ordenar el espacio interior del hospital mediante un sistema homogéneo de cartelería en offset que se presenta, ante la vista de todos, como oficial. El Gobierno de la Ciudad de Buenos Aires de a poco se hace presente en los nuevos sectores del hospital, con cartelería fácilmente identificable pero sin pretensión de exhaustividad, incompleta. En lugar de asumir las funciones comunicativas típicamente dependientes de actores estatales (como la formulación y puesta en práctica de campañas o la señalización del espacio, que quedan para privados y ONG), el objetivo parece ser la atribución del gobierno de las obras de restauración del hospital. 
La privatización del espacio semiótico presenta como limitación el paso del tiempo: las prácticas y recorridos que es posible realizar en el hospital cambian constantemente y no se observa una actualización del paisaje lingüístico por parte de los agentes privados. En su lugar, emergen respuestas espontáneas de actores institucionales que, con distintos grados de formalidad, dejan sus marcas en el paisaje lingüístico visible. Estas respuestas ocupan una posición subordinada: corrigen, complementan o actualizan, pero no dejan sin efecto las anteriores intervenciones, conformando una comunicación estratificada y jerarquizada, cada vez más confusa para los usuarios.

Como último recurso en el acceso a sus derechos está la agencia de los propios usuarios, aquello que en el paisaje no es visible pero se oye. Los mediadores, formados en el desarrollo progresivo de competencias que les permiten circular y comprender el espacio hospitalario, asumen el rol de facilitadores en el acceso a la salud. En este sentido, su rol es testimonio de la heteroglosia constitutiva del hospital, no solo por la presencia de diversas lenguas, sino también por la necesidad de mediar entre variedades del propio español.

\section{Agradecimientos}

Quisiera agradecer a los/as revisores/as anónimos/as, quienes con sus comentarios y sugerencias contribuyeron a mejorar este trabajo. 


\section{Q Bibliografía}

" Barni, Monica y Massimo Vedovelli. 2012. "Linguistic landscapes and language policies". En Linguistic landscapes, multilingualism and social change. Diversité des approaches, editado por C. Hélot, M. Barni, R. Janssens, y C. Bagna, 27-38. Frankfurt: Peter Lang.

" Blackwood, Robert, Elizabeth Lanza y Hirut Woldemariam. 2016. Negotiating and contesting identities in linguistic landscapes. Bloomsbury Publishing.

"Blommaert, Jan. 2009. "Language, asylum, and the national order". Current Anthropology 50 (4): 415-441.

" Blommaert, Jan. 2013. Ethnography, Superdiversity and Linguistic Landscapes. Chronicles of Complexity. Bristol-Buffalo-Toronto: Multilingual Matters.

» Blommaert, Jan y Ico Maly. 2014. "Ethnographic linguistic landscape analysis and social change: A case study". Tilburg Papers in Culture Studies 100: 11-33.

» Bonnin, Juan E. 2015. "Políticas lingüísticas y salud en Argentina: las campañas sanitarias plurilingües”. Trabajo presentado en el I Congreso Latinoamericano de Glotopolítica, Santiago de Chile, 13-14 de agosto.

"Bonnin, Juan E. 2018. Discourse and mental health. Voice, inequality and resistance in medical settings. Routledge.

" Briggs, Charles y Richard Bauman. 1992. “Genre, intertextuality, and social power”. Journal of linguistic anthropology 2 (2): 131-172.

»Calvet, Louis-Jean. 1997. Las políticas lingüísticas. Buenos Aires: Edicial.

»Calvi, Maria Vittoria. 2018. “Español e italiano en el paisaje lingüístico de Milán. ¿Traducción, mediación o translanguaging?”. Lingue e Linguaggi 25: 145-172.

" Courtis, Corina. 2011. "La Argentina como contexto inmigratorio: una mirada socio/etnolingüística". En Para una política del lenguaje en Argentina, editado por Lía Varela, 137-155. Caseros: EDUNTREF.

» Fernández Vavrik, Germán. 2012. "La alternancia de código como alternancia de marco: una propuesta para pensar la comunicación cotidiana con recién llegados”. En Prácticas y repertorios plurilingües en Argentina, editado por Virginia Unamuno y Angel Maldonado. Barcelona: GREIP, Grup de Recerca en Ensenyament i Interacció Plurilingües, Universitat Autònoma de Barcelona.

》 Finkelstein, Laura. 2017. "Miradas sobre usuarios migrantes regionales e interculturalidad en salud". Revista Migraciones Internacionales. Reflexiones desde Argentina 2 (1): 40-58.

"Flores, Glenn. 2005. "The impact of medical interpreter services on the quality of health care: a systematic review". Medical care research and review 62(3): 255-299.

»Gandulfo, Carolina. 2012. "'Guaraní sí, castellano más o menos': Etnografía en colaboración con niño/as en una escuela rural de Corrientes, Argentina". Spanish in context 9(2): 315-338.

» Glozman, Mara y Daniela Lauria. 2012. Voces y ecos. Una antología de los debates sobre la lengua nacional (Argentina, 1900-2000). Buenos Aires: Cabiria.

》 Goffman, Erving. 1981. Forms of talk. Philadelphia: University of Pennsylvania Press.

» Gorter, Durk. 2006. Linguistic landscape: A new approach to multilingualism. Multilingual Matters. 
» Gumperz, John J. 1982. Discourse Strategies. Cambridge: Cambridge University Press.

» Jaworski Adam y Crispin Thurlow. 2010. Semiotic Landscapes. Language, Image, Space. Bloomsbury, London: A\&C Black.

» Jelin, Elizabeth. 2006. Salud y migración regional: ciudadanía, discriminación y comunicación intercultural. Buenos Aires: IDES.

» Jewitt, Carey. 2009. The Routledge Handbook of Multimodal Analysis. Londres/ New York: Routledge.

» Kress, Gunther. 2009. Multimodality: A Social SemioticApproach to Contemporary Communication. Londres: Routledge.

» Kress, Gunther y Theo van Leeuwen. 2001. Multimodal Discourse: The modes and media of contemporary communication. Londres/ Nueva York: Oxford University Press.

» Landry, Rodrigue y Richard Bourhis. 1997. "Linguistic landscape and ethnographic vitality: An empirical study”. Journal of Language and Social Psychology 16: 23-49.

» Martín Rojo, Luisa. 2014. “Occupy: The spatial dynamics of discourse in global protest movements". Journal of Language and Politics 13(4): 583-598.

» Moustaoui, Adil. 2019. "La lengua árabe marroquí de los carteles: análisis desde los estudios del paisaje lingüístico y la política de la lengua”. Miscelánea de Estudios Árabes y Hebraicos. Sección Árabe-Islam 68: 231-262.

» Moyer, Melissa. 2011. "What multilingualism? Agency and unintended consequences of multilingual practices in a Barcelona health clinic". Journal of Pragmatics 43(5): 1209-1221.

» Park, Joseph y Mary Bucholtz. 2009. “Introduction. Public transcripts: Entextualization and linguistic representation in institutional contexts". Text \& Talk-An Interdisciplinary Journal of Language, Discourse \& Communication Studies 29(5): 485-502.

» Remorini, Carolina; Fernanda Esnaola, María Teresa Poccioni, y María Alejandra Wagner. 2007. Hospitales públicos: la mirada de usuarios y trabajadores. Estudios preliminares. Subsecretaría de Planificación de la Salud, Ministerio de Salud de la Provincia de Buenos Aires, Gobierno de la Prov. de Buenos Aires.

» Rampton, Ben. 1997. “Language Crossing and the Redefinition of Reality: Implications for Research on Code-switching community". En Code-switching in Conversation: Language, Interaction and Identity, editado por Peter Auer. London: Routledge.

» Rubdy, Rani y Selim B. Said. 2015. Conflict, exclusion and dissent in the linguistic landscape. Springer.

"Shohamy, Elana. 2015. "Linguistic landscape research as expanding language and language policy”. Linguistic Landscape 1 (1/2): 152-171.

»Shohamy, Elana y Durk Gorter. 2008. Linguistic landscape: Expanding the scenery. Routledge.

»Shohamy, Elena, Eliezer B. Rafael y Monica Barni. 2010. Linguistic landscape in the city. Multilingual Matters.

» Schuster, Michal. 2012. "Language accessibility of signage in public settings: A case study of a health care service". Southern African Linguistics and Applied Language Studies, 30 (3): 311-324.

»Schuster, Michal, Irit Elroy y Ido Elmakais. 2016. “We are lost: measuring the accessibility of signage in public general hospitals". Language Policy 16 (1): 23-38.

» Soler-Carbonell, Josep. 2016. “Complexity perspectives on linguistic landscapes. A scalar analysis”. Linguistic Landscape 2 (1): 1-25. 
»Spolsky, Bernard. 2009. Language management. Cambridge University Press.

» Unamuno, Virginia. 2016. Lenguaje y educación. Quilmes: Universidad Nacional de Quilmes.

» Unamuno, Virginia y Juan E. Bonnin. 2018. “'We work as bilinguals': Socioeconomic Changes and Language Policy for Indigenous Languages in El Impenetrable (Chaco, Argentina)". En The Oxford Handbook of Language Policy and Planning, editado por James W. Tollefson y Miguel Pérez-Milans. Oxford: Oxford University Press.

»Vilar, Milagros. 2016. “¿Qué significa 'hablar otra lengua’? Análisis interaccional de entrevistas acerca de la comunicación en el hospital”. Trabajo presentado en el XV Congreso de la Sociedad Argentina de Lingüística, Bahía Blanca, 11-14 de mayo.

»Vilar, Milagros. 2018. "Gestión de la diversidad lingüística y cultural en un hospital de Buenos Aires: un estudio de caso". Tesis de maestría, Universidad Nacional de Tres de Febrero.

» Vilar, Milagros y Luz Espain. 2016. “Trajinando en el saber médico. Narrativas de experiencia en la construcción de un saber sobre la enfermedad”. En Temas de discurso público e interacción, editado por Isolda Carranza y Mariana Cucatto, 236-26o. Bahía Blanca: EdiUNS. 